\section{ORIGINAL} RESEARCH

\section{J. Puig \\ S. Pedraza \\ A. Demchuk \\ J. Daunis-i-Estadella \\ H. Termes \\ G. Blasco \\ G. Soria \\ I. Boada \\ S. Remollo \\ J. Baños \\ J. Serena \\ M. Castellanos}

\title{
Quantification of Thrombus Hounsfield Units on Noncontrast CT Predicts Stroke Subtype and Early Recanalization after Intravenous Recombinant Tissue Plasminogen Activator
}

\begin{abstract}
BACKGROUND AND PURPOSE: Little is known about the factors that determine recanalization after intravenous thrombolysis. We assessed the value of thrombus Hounsfield unit quantification as a predictive marker of stroke subtype and MCA recanalization after intravenous rtPA treatment.
\end{abstract}

\begin{abstract}
MATERIALS AND METHODS: NCCT scans and CTA were performed on patients with MCA acute stroke within 4.5 hours of symptom onset. Demographics, stroke severity, vessel hyperattenuation, occlusion site, thrombus length, and time to thrombolysis were recorded. Stroke origin was categorized as LAA, cardioembolic, or indeterminate according to TOAST criteria. Two blinded neuroradiologists calculated the Hounsfield unit values for the thrombus and contralateral MCA segment. We used ROC curves to determine the rHU cutoff point to discriminate patients with successful recanalization from those without. We assessed the accuracy (sensitivity, specificity, and positive and negative predictive values) of $\mathrm{rHU}$ in the prediction of recanalization.
\end{abstract}

RESULTS: Of 87 consecutive patients, 45 received intravenous rtPA and only 15 (33.3\%) patients had acute recanalization. $\mathrm{rHU}$ values and stroke mechanism were the highest predictive factors of recanalization. The Matthews correlation coefficient was highest for rHU (0.901). The sensitivity, specificity, and positive and negative predictive values for lack of recanalization after intravenous rtPA for rHU $\leq$ 1.382 were $100 \%, 86.67 \%, 93.75 \%$, and $100 \%$, respectively. LAA thrombi had lower $\mathrm{rHU}$ than cardioembolic and indeterminate stroke thrombi $(P=.004)$.

CONCLUSIONS: The Hounsfield unit thrombus measurement ratio can predict recanalization with intravenous rtPA and may have clinical utility for endovascular treatment decision making.

\begin{abstract}
ABBREVIATIONS: ASPECTS = Alberta Stroke Program Early CT Score; DIAS = Desmoteplase in Acute Ischemic Stroke; DICOM = digital imaging and communication in medicine; HMCAS = hyperdense middle cerebral artery sign; ICC = intraclass correlation coefficient; IQR = interquartile range; $\mathrm{LAA}=$ large artery atherosclerosis; $\mathrm{MIP}=$ maximum intensity projection; $\mathrm{mRS}=$ modified Rankin Scale; NINDS = National Institute of Neurological Disorders and Stroke; rHU = Hounsfield Unit ratio; $r_{\varphi}=$ Matthews correlation coefficient; $\mathrm{ROC}=$ receiver operating characteristic; rt-PA = recombinant tissue plasminogen activator; TIBI = Thrombolysis in Brain Ischemia; TIMI = Thrombolysis in Myocardial Infarction; TOAST = Trial of Org 10172 in Acute Stroke Treatment
\end{abstract}

B ased on the NINDS trial, intravenous rtPA remains the only treatment approved for acute stroke. ${ }^{1}$ Early reperfusion of ischemic brain tissue is the strongest predictor of neu-

\section{Received April 13, 2011; accepted after revision August 8.}

From the Departments of Radiology (Institut de Diagnòstic per la Imatge) (J.P., S.P., H.T., G.B., S.R., J.B.) and Neurology (J.S., M.C.), Girona Biomedical Research Institute, Hospital Universitari de Girona Dr Josep Trueta, Girona, Spain; Doctoral Program (J.P.), Universitat de Barcelona, Barcelona, Spain; Department of Clinical Neurosciences (A.D.), Hotchkiss Brain Institute, University of Calgary, Calgary, Alberta, Canada; Department of Brain Ischemia and Neurodegeneration (G.S.), Institut d'Investigacions Biomèdiques de Barcelona-Consejo Superior de Investigaciones Científicas, Institut d'Investigacions Biomèdiques August Pi i Sunyer, Barcelona, Spain; Department of Computer Science and Applied Mathematics (J.D.-i.-E.) and Institute of Informatics and Applications (I.B.), Department of Informatics and Applied Mathematics and Institute of Informatics and Applications, University of Girona, Girona, Spain.

Paper previously presented at: 96th Scientific Assembly and Annual Meeting, of the Radiological Society of North America, November 28-December 3, 2010; Chicago, Illinois; and International Stroke Conference, February 9-11, 2011; Los Angeles, California.

Please address correspondence to Josep Puig, MD, Department of Radiology (Institut de Diagnòstic per la Imatge [IDI]), Girona Biomedical Research Institute, Hospital Universitari Dr Josep Trueta, Av de Francia sn. 17007, Girona, Spain; e-mail: jpuigalcantara@yahoo.es

Indicates open access to non-subscribers at www.ajnr.org

Indicates article with supplemental on-line tables.

Indicates article with supplemental on-line figure.

http://dx.doi.org/10.3174/ajnr.A2878 rologic improvement. ${ }^{2}$ Nevertheless, $\mathrm{rtPA}$ fails in $\leq 75 \%$ of patients who do not recanalize acutely or recanalize too late, leading to larger infarcts and poor clinical outcome. ${ }^{3}$

Little is known about the factors that determine the success of intravenous thrombolysis in patients with acute stroke. Age, stroke severity, systolic hypertension, hyperglycemia, HMCAS, and time to thrombolytic treatment were independent negative predictors of recanalization in multivariate regression models. ${ }^{4,5}$ Moreover, occlusion site, residual flow on transcranial Doppler at the site of occlusion, and cardioembolic stroke subtype predict the likelihood of complete recanalization. ${ }^{6-8}$ On the other hand, the presence of HMCAS, a highly specific marker of vascular occlusion, has been associated with stroke severity at baseline, but its value in predicting the efficacy of intravenous rtPA and functional outcome has been questioned. ${ }^{9-11}$

NCCT and CTA detect thrombi in large arteries and provide information about thrombus composition based on Hounsfield unit. ${ }^{12,13}$ Postmortem studies have demonstrated that thromboembolic stroke can be caused by white, red, or mixed blood cell clots. ${ }^{14}$ White cell thrombi contain varying amounts of atheromatous and cellular debris, fibrin, and 
platelets but few red cells, so they have lower Hounsfield unit counts. In contrast, red cell clots have higher concentrations of hemoglobin, so they have higher Hounsfield unit counts. ${ }^{15}$ White cell clots in the cerebral arteries are more common in patients with primary LAA than in those with thrombus from cardioembolic sources. Some studies have demonstrated that white cell clots are more resistant to thrombolytics than red cell clots. ${ }^{16}$

Noninvasive tools that may be useful for the prediction of rtPA failure would be useful for choosing other recanalization strategies, including new drugs targeting other components of the thrombus or early preparation for interventional procedures to remove clots. ${ }^{17}$ Additionally, early determination of the etiologic factors of stroke is essential for secondary prevention because the risk of recurrence is highly dependent on the underlying cause. $^{18}$

We aimed to investigate whether the success of rtPA-induced arterial recanalization can be predicted by Hounsfield unit quantification on NCCT in patients after MCA acute ischemic stroke and the potential of Hounsfield unit quantification for determining the work-up of stroke in daily practice.

\section{Materials and Methods}

\section{Patients}

We retrospectively studied MCA occlusion patients with disabling stroke symptoms treated with intravenous rtPA admitted to our hospital from December 2008 to January 2010 within 4.5 hours from symptom onset. ${ }^{19}$ Inclusion criteria were the following: 1) age from 18 to 80 years, 2) MCA occlusion documented on CTA before intravenous thrombolysis, and 3) NIHSS score at admission $>4$. Standard intravenous $\mathrm{rtPA}$ therapy was administered $(0.9 \mathrm{mg} / \mathrm{kg}$; maximum, $90 \mathrm{mg} ; 10 \%$ bolus; $90 \%$ continuous infusion). Exclusion criteria included history of serious allergic-like reactions to iodine-based contrast material, and carotid T occlusion. According to published guidelines, patients without clinical improvement after rtPA underwent mechanical thrombectomy. ${ }^{20}$ Stroke severity was assessed by the NIHSS score. ${ }^{21}$ Functional outcome was evaluated at 3 months by using mRS; patients were considered functionally independent if mRS was $\leq 2 .{ }^{22}$ Stroke mechanism was classified by using the modified TOAST criteria. ${ }^{23}$ The analysis by subtypes was confined to 3 major stroke subtypes: LAA, cardioembolic, and indeterminate cause of stroke. The local ethics committee approved the study protocol.

\section{Imaging Protocols}

We used a 16-detector CT scanner (MX8000 IDT; Philips Healthcare, Best, the Netherlands) to perform standard brain studies with the following parameters: axial nonhelical scan acquisition with collimation, $0.75 \mathrm{~mm}$; tube current, $350 \mathrm{mAs}$; tube voltage, $120 \mathrm{kV}$; time cycle, 2; rotation speed, 0.75 seconds; standard resolution; section thickness, $3 \mathrm{~mm}$; and matrix, $512 \times 512$. We obtained continuous axial sections parallel to the orbitomeatal line from the base of the skull to the vertex.

CTA was performed with low-osmolar nonionic monomer iopromide (Ultravist; Bayer Schering Pharma, Berlin, Germany) at $300 \mathrm{mg}$ iodine/mL. Contrast-enhanced image acquisition was initiated by visual contrast bolus tracking in the cervical arteries after an $80-\mathrm{mL}$ bolus was infused at a rate of $4 \mathrm{~mL} / \mathrm{s}$, followed by $20 \mathrm{~mL}$ of saline solution at $4 \mathrm{~mL} / \mathrm{s}$. The vessels were scanned in the helical mode with the following parameters: collimation, $16 \times 0.75$; tube current, 300
mAs; tube voltage, $120 \mathrm{kV}$; rotation speed, 0.75 seconds; and pitch, 0.98 . Reconstruction parameters for the axial sections were the following: effective section thickness, $1 \mathrm{~mm}$; increments, $0.5 \mathrm{~mm}$; reconstruction kernel filter, C; and matrix, $512 \times 512$.

Carotid and transcranial duplex sonography were performed in all patients, and MCA was monitored during rtPA perfusion. When CTA was performed 1 hour after thrombolysis, the protocol was the same as that at admission. Finally, 1.5T MR imaging (Intera, Philips Healthcare) was performed within 12 hours of onset; the protocol included axial trace DWI, FLAIR, gradient-echo sequences, and perfusion-weighted imaging. Postperfusion intracranial MRA was performed by using a standard method. ${ }^{24}$

\section{Image Analysis}

All DICOM data were transferred to Starviewer software, a platform developed in our research laboratory (http://gilab.udg.edu/). The HMCAS was defined by the following criteria ${ }^{25}: 1$ ) spontaneous visibility of the whole horizontal part of the MCA, 2) attenuation of the MCA higher than that of the surrounding brain, 3) disappearance on bone windows, 4) unilaterality, and 5) absence of subarachnoid hemorrhage. The M2 dot sign was defined as hyperattenuation of an arterial structure in the Sylvian fissure relative to the contralateral side. ${ }^{26}$ On the basis of CTA images, ROIs were manually placed on the thrombus and on the contralateral MCA on NCCT by 2 independent neuroradiologists (J.P., H.T.) blinded to clinical data. The Hounsfield unit values were obtained for each ROI by averaging all voxels within the ROI. The final Hounsfield unit value for each thrombus was calculated by summing the Hounsfield unit of the ROIs in each section in which the thrombus was seen and dividing by the number of sections (Fig 1). Because thrombus Hounsfield unit counts are affected by the hematocrit, ${ }^{13}$ they were corrected by determining the Hounsfield unit of the contralateral normal segment and calculating the ipsilateral-tocontralateral $\mathrm{rHU}$ (rHU = MCA symptomatic side / MCA asymptomatic side). Two raters (J.P., H.T.) determined the Hounsfield unit independently, and their results were averaged. Additionally, 1 rater (J.P.) repeated Hounsfield unit measurements in all patients 1 month after the first determination. We calculated the intraobserver and interobserver reliabilities.

To determine the true extent of vascular occlusion, CTA images were obtained before rtPA. The thrombus length was determined on the axial thick-slab MIP image of the CTA dataset. We assessed the patency of the MCA with the modified TIMI grading system, as follows ${ }^{27}$ : complete occlusion with no perfusion past the thrombus (TIMI 0); perfusion past the initial occlusion with no opacification of the distal vessels (TIMI 1); partial occlusion (TIMI 2), defined as an obstruction with opacification of the distal vessels; and complete recanalization (TIMI 3). Standard (TIBI) grading $(0-5)$ was used to score vessel flow on transcranial duplex sonography. Recanalization status was immediately determined by certified staff by using transcranial duplex sonography (J.S., M.C.) and from 1 hour after thrombolysis by using CTA or MRA. Recanalization was defined as TIBI 3-5 or TIMI 2-3 flow in vessels. ${ }^{28}$ TIBI and TIMI scores have good correlation. ${ }^{3}$ If the 2 observers' scores differed, the final decision was reached by consensus.

\section{Statistical Analysis}

Descriptive statistics were expressed as a median with a range for continuous variables and as numbers (percentages) for categoric variables. We compared baseline data, clinical variables at admission, stroke etiology, results of neurologic assessment, other CT findings, 


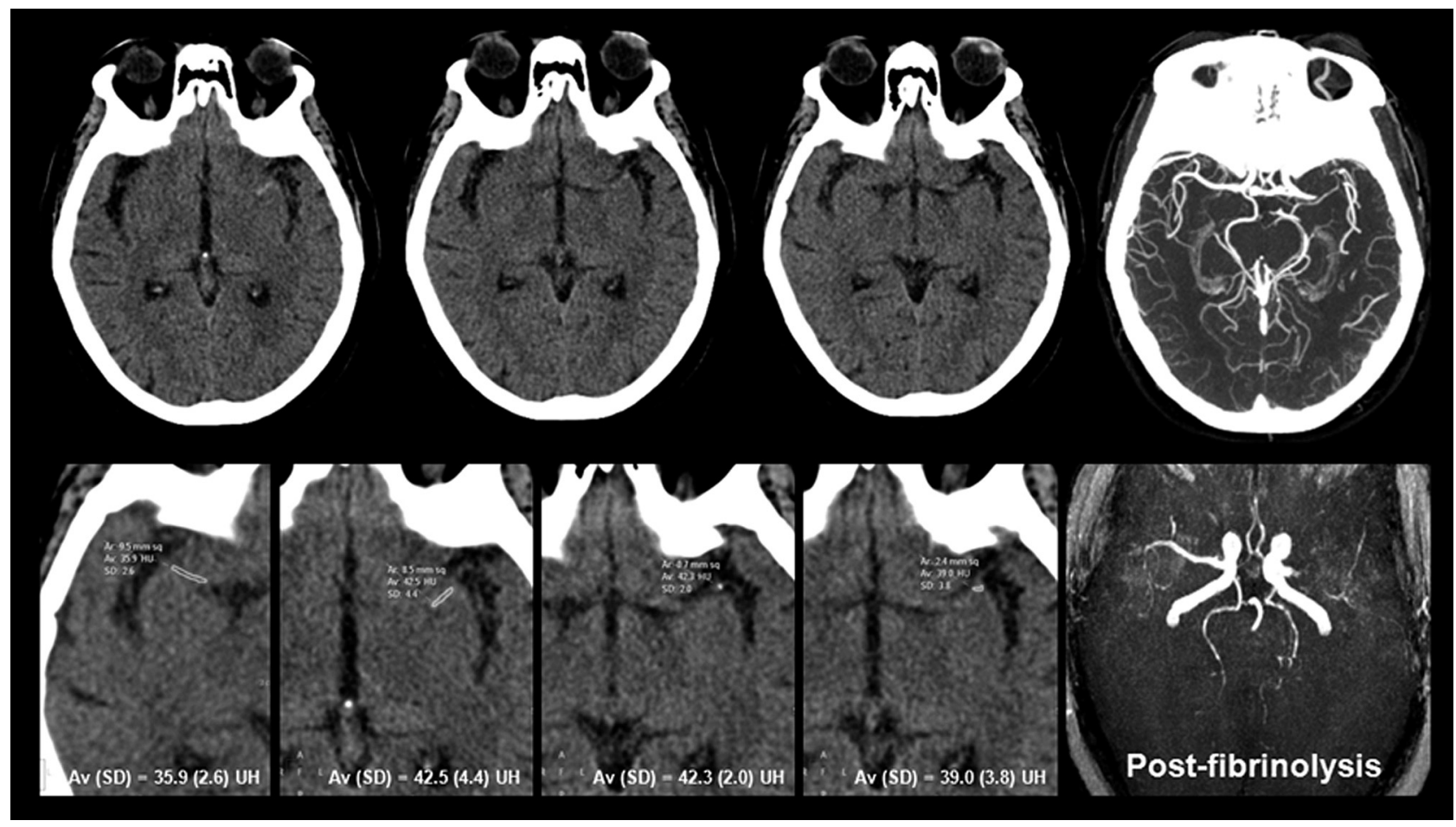

Fig 1. A 78-year-old woman with severe right-sided hemiparesis and aphasia 70 minutes after symptom onset. NCCT demonstrates left HMCAS involving the proximal segment (M1) of the MCA. The rHU of the thrombus was 1.14 (ROI values at bottom row on magnified zoom $\times 3$ ). Intravenous rtPA failed to achieve recanalization.

and outcomes between patients with complete recanalization and patients with persistent occlusion. We used multivariate analyses to identify prognostic factors for MCA recanalization after rtPA and to determine the prognostic significance of rHU for recanalization. For univariate comparison between patients with recanalization and those without, we used the $t$ test for numeric data and the $\chi^{2}$ test (with Yates correction for $2 \times 2$ tables or, in case of small expected frequencies, the Fisher exact test) for proportions. We used multiple logistic regression to define independent predictors of functional outcome. To calculate the rHU cutoff point to discriminate recanalization and persistent occlusion, we used ROC curves. We used the $r_{\varphi}$ to determine which variables were more strongly associated with recanalization. ${ }^{29}$ Statistical significance was set at $P<.05$. To compare Hounsfield unit measurements (intraobserver and interobserver reliabilities), we used ICCs. The level of intra- and interobserver consistency was respectively classified as fair (ICC $=0.5-0.7)$, good $(0.7-$ $0.9)$, or almost perfect $(>0.90)$. All statistical evaluations were performed by using Minitab, Version 15.1.0.0 (Minitab, State College, Pennsylvania).

\section{Results}

\section{Subjects}

Among 87 consecutive patients admitted for acute MCA ischemic stroke, 39 (44.7\%) were excluded from the study because they did not meet 1 of the following study inclusion/exclusion criteria: contraindication for iodine-based contrast material injection resulting in no CTA performed ( $n=4,4.6 \%)$, no visible occlusion $(n=21,24 \%)$, NIHSS score $\leq 4(n=10$, $11.5 \%)$, and carotid T occlusion $(n=4,4.6 \%)$. Of the subjects meeting the criteria, 3 were excluded from analysis as a result of poor image quality due to motion artifacts. Thus, 45 pa- tients ( 20 women; median age, 73 years) treated with intravenous thrombolysis were available for imaging analysis.

\section{Clinical and Neuroimaging Characteristics}

On-line Table 1 summarizes patients' demographic data, riskfactor profile, and clinical and radiologic findings. The baseline median NIHSS score was 16. CTA was the technique to confirm MCA occlusion in all baseline studies. The median time from symptom onset to initiation of intravenous rtPA was $169 \pm 102$ minutes. The HMCAS and MCA dot sign were seen in 26 patients $(57.8 \%)$ and 8 patients (17.8\%), respectively. Interobserver reliability for the detection of HMCAS was substantial $(\kappa=0.70)$, and intraobserver reliability was good (ICC $=0.72)$. Stroke etiologic subtypes were LAA in 15 cases $(33.3 \%)$, cardioembolic in $21(46.7 \%)$, and indeterminate in 9 (20\%). The median time from symptom onset to assessment of recanalization by follow-up vascular imaging was $305 \pm 115$ minutes. Follow-up vascular imaging was CTA in 18 (40\%) subjects, MRA in $27(60 \%)$ subjects, and transcranial duplex sonography in $41(91.1 \%)$ subjects. If available, recanalization was assessed by CTA, and if not, by MRA.

M1 occlusion was present in $57.8 \%$ of cases. Recanalization was achieved in 15 patients (33.2\%). M2 occlusions were present in 11 patients $(24.3 \%)$, and recanalization was achieved in $6(54.6 \%)$ cases (Fig 2). The rate of persistent occlusion was more frequent in LAA $(94 \%)(P=.007)$. The rate of persistent occlusion was $52.4 \%$ in cardioembolic etiologies and $55.6 \%$ in indeterminate causes.

Among the occlusion sites, best outcomes were achieved for M1-MCA (53\%), followed by M2-MCA (40\%), and M1-M2 MCA (7\%). Only 5 of the patients in whom rtPA 


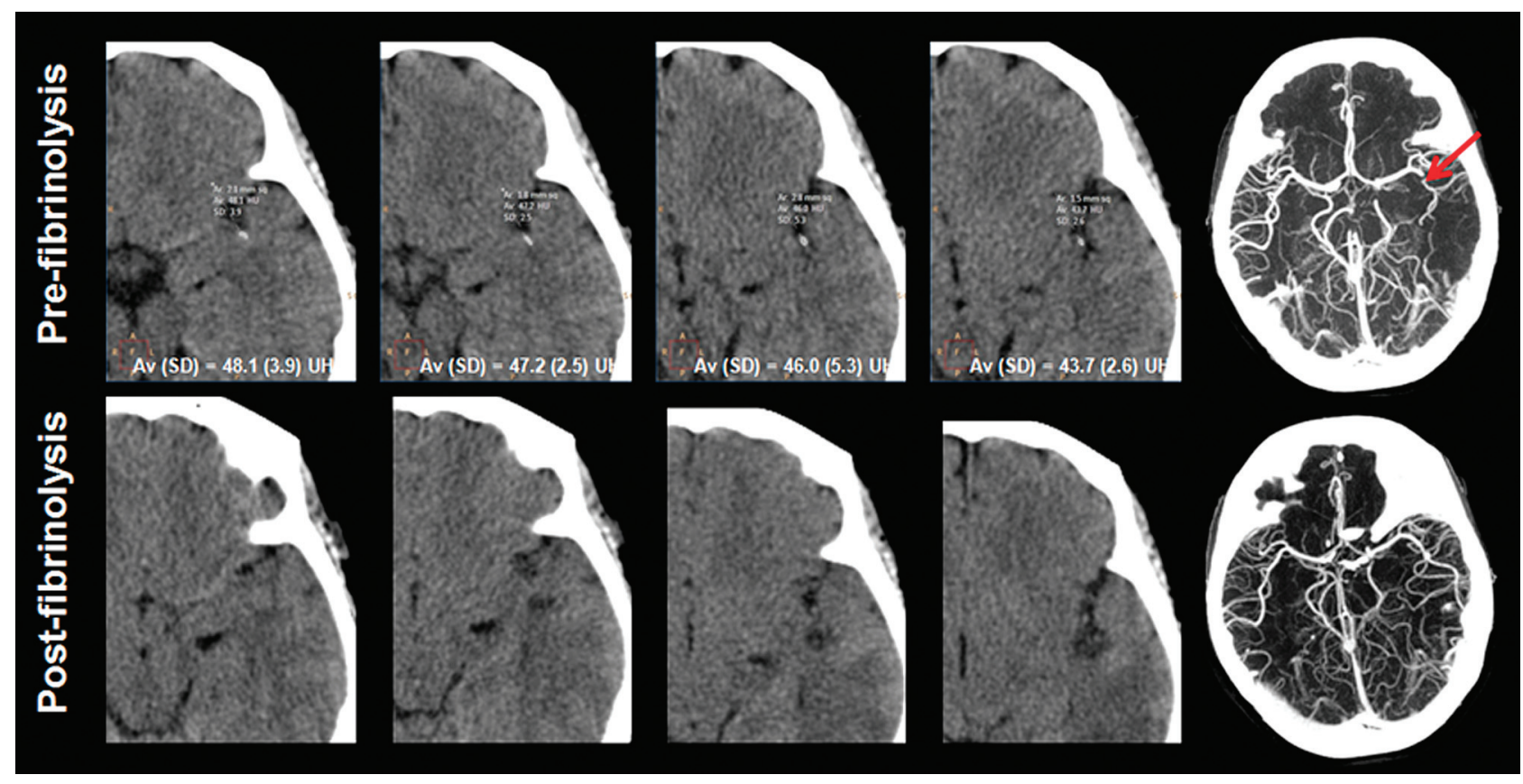

Fig 2. A 64-year-old man with aphasia 180 minutes after symptom onset. NCCT demonstrates a hyperattenuated thrombus in the left M2 segment, corroborated on CTA axial MIP (arrow). The rHU of the thrombus was 1.48. Treatment succeeded, and the vascular hyperattenuation disappeared (bottom row).

failed to recanalize the occluded artery underwent mechanical thrombectomy because this treatment was unavailable until the last 2 months of this study.

\section{Analysis of Variables Associated with Early Recanalization}

On-line Table 1 reports the univariate comparison of the recanalized and unrecanalized subgroups. Absolute Hounsfield unit, contralateral MCA Hounsfield unit, rHU, and LLA were the variables associated to recanalization; rHU was the strongest predictor of recanalization $\left(r_{\varphi}=0.901\right)$ (On-line Table 2$)$. Mean rHUs of recanalized and unrecanalized thrombi were $1.57 \pm 0.23$ and $1.11 \pm 0.15$, respectively $(P<.001, T$ value $=$ $6.81)$. The estimated interobserver and intraobserver reliabilities showed good agreement for Hounsfield unit measurements (intraclass correlation coefficients were 0.745 and 0.801 , respectively).

We calculated the rHU cutoff value with the highest predictive capacity for persistent occlusion. This analysis showed that $\mathrm{rHU}<1.382$ predicted persistent occlusion after intravenous rtPA with a sensitivity, specificity, and positive and negative predictive values of $100 \%, 86.67 \%, 93.75 \%$, and $100 \%$, respectively (Fig 3).

The HMCAS was present in $9(60 \%)$ recanalized thrombi, and 5 of these had rHU $>1.382$. The HMCAS was present in 17 (65.39\%) unrecanalized thrombi, and all had $\mathrm{rHU}<1.382$. Thrombi were isoattenuated or hypoattenuated in 12 cases (26.67\%); $9(75 \%)$ of these had $\mathrm{rHU}<1.382$ and did not recanalize (Fig 4 and On-line Fig. 1).

The mean absolute Hounsfield unit values were $42.093 \pm$ 4.09 for unrecanalized and $49.330 \pm 7.07$ for recanalized thrombi $(P=.001, T$ value $=3.77)$. The sensitivity, specificity, and positive and negative predictive values for persistent occlusion after intravenous rtPA for absolute Hounsfield

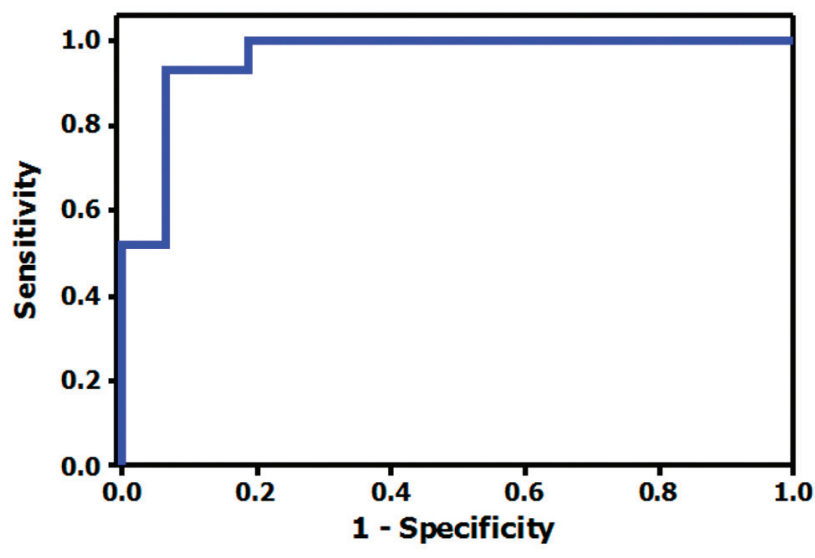

Fig 3. ROC curve for Hounsfield unit ratios in the prediction of vascular recanalization. The area under the ROC curve is equal to 0.96 .

units $<50.90$ were $100 \%, 37.50 \%, 81.81 \%$, and $100 \%$, respectively. The area under the ROC curve was 0.79 .

The contralateral MCA Hounsfield unit mean values were $36.721 \pm 2.75$ for unrecanalized and $32.470 \pm 6.41$ for recanalized thrombi $(P=.021, T$ value $=-2.53)$. The area under the ROC curve was 0.76 .

Although rHU values, absolute thrombus Hounsfield unit values, and contralateral MCA values were predictive factors of recanalization, both the $T$ value, with equivalent degrees of freedom, and the area under the ROC curve are better for the rHU values. It was not possible to include all the Hounsfield unit values, relative and absolutes, due to the multicollinearity between them.

\section{Hounsfield Unit Quantification on Thrombus and Stroke Subtype}

The HMCAS was present in $73.2 \%$ of LAA, in $42.9 \%$ of cardioembolic, and in $55.6 \%$ of indeterminate thrombi. The rHU 

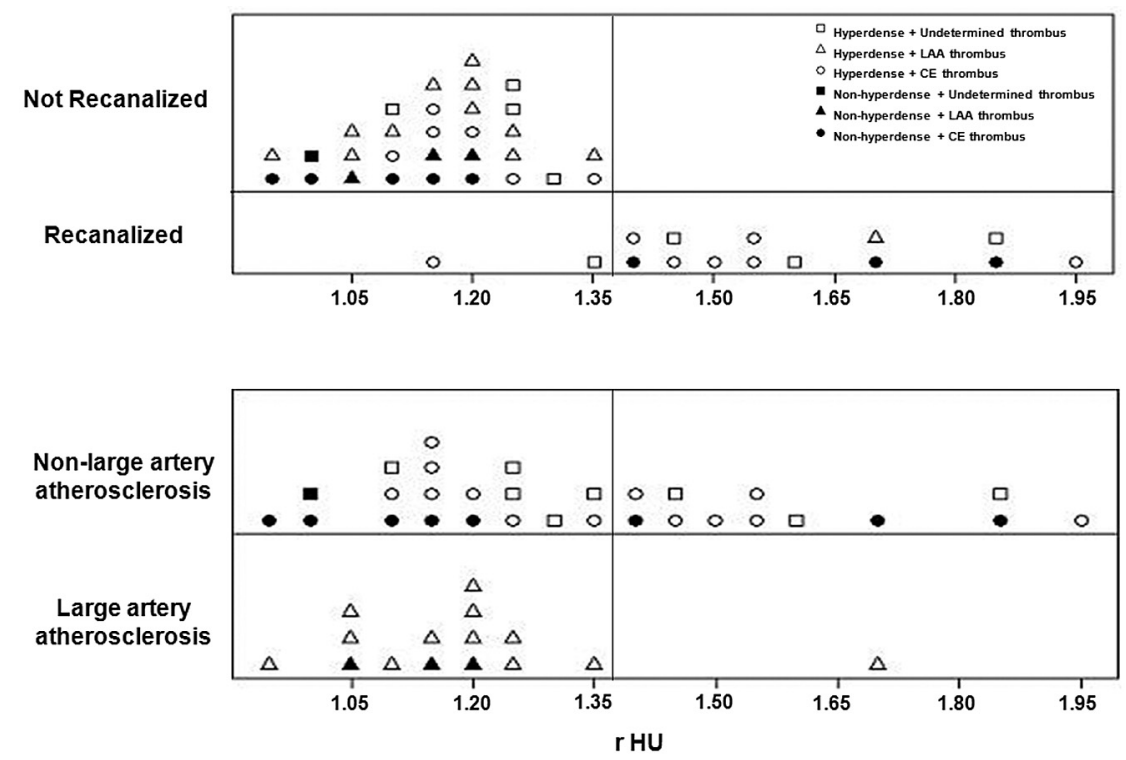

Fig 4. Distribution of thrombi according to the effectiveness of intravenous rtPA and stroke etiology. The cutoff value is set at 1.382 to cover all thrombi that failed to recanalize after intravenous rtPA (top). Values above this cutoff point can reliably discriminate thrombi from LAA (above). CE indicates cardioembolic.

values were $1.372 \pm 0.31$ for cardioembolic stroke, $1.101 \pm$ 1.17 for LAA stroke, and $1.462 \pm 0.26$ for stroke of indeterminate origin (On-line Table 1 ). We found $\mathrm{rHU}<1.382$ in $93.3 \%$ of LAA thrombi $(P=.020)$ and in $57.1 \%$ of cardioembolic thrombi ( $P=.053$ ) (Fig 4 ). The sensitivity, specificity, and positive and negative predictive values of $\mathrm{rHU} \leq 1.382$ for LAA stroke subtype were $93.3 \%, 40 \%, 43.6 \%$, and $92.3 \%$, respectively.

\section{Discussion}

Our study shows that Hounsfield unit quantification of thrombus in CTA-proved MCA proximal occlusion can predict the success of intravenous rtPA recanalization. Although significant differences were noted when absolute thrombus and contralateral Hounsfield unit values were analyzed, rHU was the strongest predictor of recanalization. Specifically, a cutoff of rHU $<1.382$ derived by ROC analysis was associated with nonrecanalization after intravenous rtPA treatment in acute ischemic stroke. This method might be useful for rapid triage of patients for thrombolytic therapy to decide which patients should undergo endovascular rescue therapy.

Our findings are in good agreement with those of Kim et al, ${ }^{13}$ who found that CT-based measurement of Hounsfield units may be helpful in predicting the effectiveness of thrombolytic therapy. Hemoglobin determines the attenuation of a clot, and lysability appears to increase with increasing hematocrit levels, suggesting that the composition of the thrombus determines its susceptibility to intravenous thrombolysis. ${ }^{12,13}$ White thrombi consist of varying amounts of platelets, atheromatous, and cellular debris, with few red cells, resulting in lower Hounsfield unit values and greater resistance to fibrinolytic agents. Red thrombi contain erythrocytes and some fibrin, resulting in higher Hounsfield unit counts and greater susceptibility to fibrinolytic agents. ${ }^{15,16}$ Experimental studies show that white thrombi retract more than red thrombi, resulting in reduced permeability to the bulk flow of thrombo- lytic agents, increased fibrin content per unit of clot volume, and decreased plasminogen content. ${ }^{16,30}$

The HMCAS is an indicator of intraluminal thrombus; this early CT finding is reported in $5 \%-59 \%$ of acute ischemic strokes. ${ }^{10-12,31,32}$ Most interesting, we found that a hyperattenuated thrombus does not necessarily have high Hounsfield unit values. Perceiving low-attenuation thrombi as hyperattenuated could be due to the limited sensitivity of the human eye to shades of gray. We found $\mathrm{rHU}<1.382$ in more than half of the HMCASs seen. The efficacy of intravenous rtPA in patients with the HMCAS and its impact in terms of functional outcome remain controversial. ${ }^{10,11,25}$ A systematic review of early CT signs of stroke revealed that the HMCAS was associated with worse functional outcome at 6 months. ${ }^{4}$ Nevertheless, the NINDS and the European Cooperative Acute Stroke Study found better outcomes when patients with HMCAS were treated with intravenous rtPA compared with placebo, and the HMCAS was not an independent predictor of poor outcome. ${ }^{11,33}$ The HMCAS was more prevalent in our population than in other series, probably because the thin sections ( $3 \mathrm{~mm}$ ) that we obtained on NCCT scans reduced partial volume averaging and enabled more precise visualization and delineation of small thrombi in nonlinear arteries compared with previous studies, which used section thicknesses of $\geq 5$ $\mathrm{mm} .^{10,34}$ The fact that nearly half of the thrombi in our study were attributable to cardioembolic disease may explain the increase in the rate of hyperattenuated thrombi.

Early recanalization has been associated with good clinical outcome. ${ }^{3,35}$ Our analysis suggests that rHU quantification is a better predictor of recanalization than the visual assessment of attenuation. However, we did not find worse functional outcome or higher mortality in patients in whom recanalization failed or in those with low thrombus rHU values, probably due to our small sample size. Additionally, though the thrombi from LAA had a low MCA recanalization compared with those with cardioembolic causes, this difference did not reach signif- 
icance. We excluded $\mathrm{T}$ occlusions because they are reportedly more resistant to intravenous thrombolysis than isolated MCA occlusions. ${ }^{5}$ Our findings are in line with those of Molina et $\mathrm{al},{ }^{8}$ who found more successful early rtPA recanalization in cardioembolic strokes (59\%) and low recanalization rates in patients with concomitant ipsilateral carotid artery disease.

The structure of the thrombi has an influence on their susceptibility to lysis and the penetration of thrombolytic agents compared with atherosclerotic thrombi. ${ }^{36}$ Cardioembolic thrombi are formed under conditions of relative stasis. These clots are more uniform and contain more fibrin and red cells than thrombi in LAA, so given the high binding affinity of rtPA for fibrin, the thrombolytic agent penetrates and distributes homogeneously, making successful recanalization more likely. In contrast, the ability of rtPA to penetrate old wellorganized platelet-rich clots is limited, and this limitation may result in nonuniform clot softening and less successful recanalization. ${ }^{8}$ Effective distribution of rtPA within the clot accelerates fibrinolysis, and the fibrinolytic rate depends on the pressure gradient to which the clot is exposed. ${ }^{37}$ Moreover, severe carotid artery disease might decrease the pressure gradient on the clot and hamper the delivery of rtPA into the MCA thrombus, which may explain the poor response to lysis. ${ }^{8,37,38}$

Several limitations warrant consideration in this study. The study was small in size and retrospective in design. Different imaging modalities were used to determine recanalization. Manual ROI placement may be subject to operator bias. Recently developed semiautomatic methods might enable quantitative thrombus analysis and thereby mitigate this potential error in the hyperacute setting without delaying treatment. ${ }^{31}$ Finally, thinner reconstructions of NCCT data with a section width of $<3 \mathrm{~mm}$ might allow the accurate measurement of Hounsfield unit quantification in the cerebral artery.

\section{Conclusions}

rHU measurement on NCCT may be useful in predicting the effectiveness of intravenous rtPA. An rHU cutoff below 1.382 may predict persistent occlusion after intravenous thrombolysis. Larger prospective studies are needed to validate these findings.

\section{Acknowledgments}

We thank John Giba for English assistance.

Disclosures: Salvador Pedraza-UNRELATED: Board Membership: Stroke journal, Comments: Member of the Editorial Board of Stroke journal; American Hospital Association; Consultancy. DIAS Trial, Comments: Member of the imaging committee of DIAS; the sponsor is the Lundbeck. Andrew Demchuk-UNRELATED: Grants/Grants Pending. NovoNordisk Canada, Payment for Lectures (including service on Speakers Bureaus): Merck, Boehringer Ingelheim, Payment for Development of Educational Presentations: Boehringer Ingelheim; Travel/Accommodations/Meeting Expenses Unrelated to Activates Listed: Boehringer Ingelheim, Comments: Travel to European Stroke Conference 2009; Stock/Stock Options: Calgary Scientific Inc, Comments: shareholder

\section{References}

1. Tissue plasminogen activator for acute ischemic stroke: the National Institute of Neurological Disorders and Stroke rt-PA Stroke Study Group. NEngl J Med 1995;333:1581-87

2. Bang OY, Liebeskind DS, Buck BH, et al. Impact of reperfusion after 3 hours of symptom onset on tissue fate in acute cerebral ischemia. $J$ Neuroimaging. 2009;19:317-22

3. Bhatia R, Hill MD, Shobha N, et al. Low rates of acute recanalization with intravenous recombinant tissue plasminogen activator in ischemic stroke: real-world experience and a call for action. Stroke 2010;41:2254-58

4. Wardlaw JM, Mielke O. Early signs of brain infarction at CT: observer reliability and outcome after thrombolytic treatment—systematic review. Radiology 2005;235:444-53

5. del Zoppo GJ, Poeck K, Pessin MS, et al. Recombinant tissue plasminogen activator in acute thrombotic and embolic stroke. Ann Neurol 1992;32:78-86

6. Saqqur M, Uchino K, Demchuk AM, et al. Site of arterial occlusion identified by transcranial Doppler predicts the response to intravenous thrombolysis for stroke. Stroke 2007;38:948-54

7. Saqqur M, Tsivgoulis G, Molina CA, et al. Residual flow at the site of intracranial occlusion on transcranial Doppler predicts response to intravenous thrombolysis: a multi-center study. Cerebrovasc Dis 2009;27:5-12

8. Molina CA, Montaner J, Arenillas JF, et al. Differential pattern of tissue plasminogen activator-induced proximal middle cerebral artery recanalization among stroke subtypes. Stroke 2004;35:486-90

9. Abul-Kasim K, Brizzi M, Petersson J. Hyperdense middle cerebral artery sign is an ominous prognostic marker despite optimal workflow. Acta Neurol Scand 2010;122:132-39

10. Tomsick T, Brott T, Barsan W, et al. Prognostic value of the hyperdense middle cerebral artery sign and stroke scale score before ultraearly thrombolytic therapy. AJNR Am J Neuroradiol 1996;17:79-85

11. Manelfe C, Larrue V, von Kummer R, et al. Association of hyperdense middle cerebral artery sign with clinical outcome in patients treated with tissue plasminogen activator. Stroke 1999;30:769-72

12. Kim EY, Lee SK, Kim DJ, et al. Detection of thrombus in acute ischemic stroke: value of thin-section noncontrast-computed tomography. Stroke 2005; 36:2745-47

13. Kim EY, Heo JH, Lee SK, et al. Prediction of thrombolytic efficacy in acute ischemic stroke using thin-section noncontrast CT. Neurology 2006;67: $1846-48$

14. Castaigne $\mathrm{P}$, Lhermitte F, Gautier JC, et al. Internal carotid artery occlusion: a study of 61 instances in 50 patients with post-mortem data. Brain 1970; 93:231-58

15. Kirchhof K, Welzel T, Mecke C, et al. Differentiation of white, mixed, and red thrombi: value of CT in estimation of the prognosis of thrombolysis phantom study. Radiology 2003;228:126-30

16. Jang IK, Gold HK, Ziskind AA, et al. Differential sensitivity of erythrocyte-rich and platelet-rich arterial thrombi to lysis with recombinant tissue-type plasminogen activator: a possible explanation for resistance to coronary thrombolysis. Circulation 1989;79:920-28

17. Nogueira RG, Schwamm LH, Hirsch JA. Endovascular approaches to acute stroke. Part 1. Drugs, devices, and data. AJNR Am J Neuroradiol 2009; 30:649-61

18. Lovett JK, Coull AJ, Rothwell PM. Early risk of recurrence by subtype of ischemic stroke in population-based incidence studies. Neurology 2004;62:569-73

19. Lansberg MG, Bluhmki E, Thijs VN. Efficacy and safety of tissue plasminogen activator 3 to 4.5 hours after acute ischemic stroke: a metaanalysis. Stroke 2009; $40: 2438-41$

20. Adams HP Jr, del Zoppo G, Alberts MJ, et al. Guidelines for the early management of adults with ischemic stroke: a guideline from the American Heart Association/American Stroke Association Stroke Council, Clinical Cardiology Council, Cardiovascular Radiology and Intervention Council, and the Atherosclerotic Peripheral Vascular Disease and Quality of Care Outcomes in Research Interdisciplinary Working Group - the American Academy of Neurology affirms the value of this guideline as an educational tool for neurologists. Stroke 2007;38:1655-711

21. Brott T, Adams HP Jr, Olinger CP, et al. Measurements of acute cerebral infarction: a clinical examination scale. Stroke 1989;20:864-70

22. Sulter G, Steen C, De Keyser J. Use of the Barthel index and modified Rankin scale in acute stroke trials. Stroke 1999;30:1538-41

23. Adams HP Jr, Bendixen BH, Kappelle LJ, et al. Classification of subtype of acute ischemic stroke: definitions for use in a multicenter clinical trial TOAST Trial of Org 10172 in Acute Stroke Treatment. Stroke 1993;24:35-41

24. Pedraza S, Silva Y, Mendez J, et al. Comparison of preperfusion and postperfusion magnetic resonance angiography in acute stroke. Stroke 2004; 35:2105-10

25. Leys D, Pruvo JP, Godefroy O, et al. Prevalence and significance of hyperdense middle cerebral artery in acute stroke. Stroke 1992;23:317-24

26. Barber PA, Demchuk AM, Hudon ME, et al. Hyperdense sylvian fissure MCA "dot" sign: a CT marker of acute ischemia. Stroke 2001;32:84-88

27. Williams DO, Borer J, Braunwald E, et al. Intravenous recombinant tissue-type plasminogen activator in patients with acute myocardial infarction: a report from the NHLBI Thrombolysis in Myocardial Infarction Trial. Circulation 1986;73:338-46

28. Demchuk AM, Burgin WS, Christou I, el al. Thrombolysis in brain ischemia (TIBI) transcranial Doppler flow grades predict clinical severity, early recovery, and mortality in patients treated with intravenous tissue plasminogen activator. Stroke 2001;32:89-93

29. Baldi P, Brunak S, Chauvin Y, et al. Assessing the accuracy of prediction algorithms for classification: an overview. Bioinformatics 2000;16:412-24 
30. Blinc A, Kennedy SD, Bryant RG, et al. Flow through clots determines the rate and pattern of fibrinolysis. Thromb Haemost 1994;71:230-35

31. Kim EY, Yoo E, Choi HY, et al. Thrombus volume comparison between patients with and without hyperattenuated artery sign on CT. AJNR Am J Neuroradiol 2008;29:359-62

32. Von Kummer R, Meyding-Lamade U, Forsting M, et al. Sensitivity and prognostic value of early CT in occlusion of the middle cerebral artery trunk. AJNR Am J Neuroradiol 1994;15:9-15

33. Qureshi AI, Ezzeddine MA, Nasar A, et al. Is intravenous tissue plasminogen activator beneficial in patients with hyperdense artery sign? Neurology 2006;66:1171-74

34. Riedel CH, Jensen U, Rohr A, et al. Assessment of thrombus in acute middle cerebral artery occlusion using thin-slice nonenhanced computed tomography reconstructions. Stroke 2010;41:1659-64
35. Khatri P, Abruzzo T, Yeatts SD, et al. Good clinical outcome after ischemic stroke with successful revascularization is time-dependent. Neurology 2009;73:1066-72

36. Blinc A, Keber D, Lahajnar G, et al. Lysing patterns of retracted blood clots with diffusion or bulk flow transport of plasma with urokinase into clots: a magnetic resonance imaging study in vitro. Thromb Haemost 1992; 68:667-71

37. Blinc A, Keber D, Lahajnar G, et al. Magnetic resonance imaging of retracted and nonretracted blood clots during fibrinolysis in vitro. Haemostasis 1992;22:195-201

38. Truilaas P, Noghoghossian N, Derex L, et al. Thrombolysis with intravenous rtPA in a series of 100 cases of acute ischemic carotid territory stroke: determination of etiological, topographic and radiological outcome factors. Stroke 1998;29:2529-40 